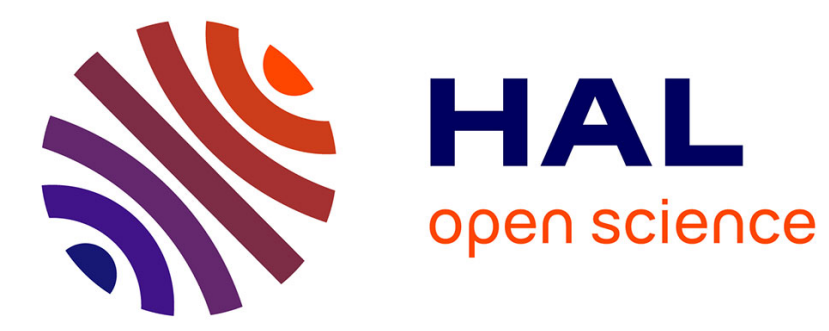

\title{
Inversion of a new circular-arc Radon transform for Compton tomography
}

\author{
M.K. Nguyen, T. T. Truong
}

\section{To cite this version:}

M.K. Nguyen, T. T. Truong. Inversion of a new circular-arc Radon transform for Compton tomography. Inverse Problems, 2010, 26, pp.065005. 10.1088/0266-5611/26/6/065005 . hal-00671806

\section{HAL Id: hal-00671806 https://hal.science/hal-00671806}

Submitted on 18 Feb 2012

HAL is a multi-disciplinary open access archive for the deposit and dissemination of scientific research documents, whether they are published or not. The documents may come from teaching and research institutions in France or abroad, or from public or private research centers.
L'archive ouverte pluridisciplinaire HAL, est destinée au dépôt et à la diffusion de documents scientifiques de niveau recherche, publiés ou non, émanant des établissements d'enseignement et de recherche français ou étrangers, des laboratoires publics ou privés. 


\title{
Inversion of a new circular-arc Radon transform for Compton scattering tomography
}

\author{
M. K. Nguyen $\ddagger$ and T. T. Truong $\dagger \dagger$ \\ ‡ Laboratoire Equipes Traitement de l'Information et Systèmes, \\ (CNRS UMR 8051/ENSEA/Université de Cergy-Pontoise), \\ 2 av. Adolphe Chauvin 95302 Cergy-Pontoise, France. \\ † Laboratoire de Physique Théorique et Modélisation \\ (CNRS UMR 8089/Université de Cergy-Pontoise), \\ 2 av. Adolphe Chauvin 95302 Cergy-Pontoise, France.
}

Abstract. A new circular arc Radon transform arising from the mathematical modeling of image formation in a new modality of Compton scattering tomography is introduced. We describe some of its properties and establish its analytic inverse formula. This result demonstrates the feasibility of image reconstruction from Compton scattered radiation in Compton scattering tomography. We also show that it belongs to a larger class of Radon transforms on algebraic curves, which remain invariant under a specific geometric inversion.

PACS numbers: 00.00, 20.00, 42.10

Submitted to: Inverse Problems

† To whom correspondence should be addressed (truong@u-cergy.fr) 


\section{Introduction}

Since the seminal work of J Radon [1], which finds numerous applications (Computed Tomography, Single Photon Emission Computed Tomography (SPECT) and Positron Emission Tomography (PET), etc.) many extensions of this integral transform have been widely discussed, in particular in the literature of imaging science. This is the case when results of measurements appear under the form of integrals of a physical quantity over lower dimensional manifolds. The relevant problem to solve is the recovery of the physical quantity of interest as a function in $\mathbb{R}^{2}$. The field of such problems is known in mathematics as integral geometry in the sense of I M Gel'fand [2] and in image processing as image reconstruction.

As the circle is the simplest non-trivial curve in the plane next to the straight line, it becomes the natural object on which a new Radon transform can be defined. However a circle has three parameters (two for its center and one for its radius). Therefore to reduce data redundancy, a constraint is usually imposed. A few examples of circular Radon transforms are known so far. Quinto [3] has considered the case of all translations of a circle of fixed radius as well as circles centered on a circle and shown that they are invertible. This case is of interest for thermo-acoustic (and opto-acoustic) tomography [4, 5]. Synthetic Aperture Radar - SAR (or SOund NAvigation and Ranging - SONAR) imaging makes use of circles of varying radius but centered on a straight line $[6,7,8]$. Circular Radon transforms along paths that are not on the zero sets of harmonic polynomials were proved to be invertible in [9]. Finally Compton scattering tomography, a two-dimensional imaging process based on scattered radiation, has one modality for which image formation is built on Radon transforms defined on a set of circles passing through a fixed point of the plane [10].

In this work we describe a recently proposed modality of Compton scattering tomography [11], which is at the origin of a new Radon transform. It is defined on circular arcs having a chord of fixed length rotating around its middle point. Obviously such a condition is far more complicated that the two conditions previously met in imaging systems. Yet, it will be shown that its inversion can be achieved through analytic techniques established long ago by A M Cormack [12].

Generally the choice of a family of circles is dictated by the physical mechanism of image formation. But there exists also other mechanisms which lead to Radon transforms on other types curves such as ellipses [13], parabolas [14], hyperbolas [15, 16] or V-line (or pair of half-lines from a vertex in the plane) [17]. It is not known whether higher order algebraic curves could support more sophisticated image formation mechanisms.

From the mathematical point of view, it was A M Cormack who first succeeded to invert a large class of Radon transform on what he called $\alpha$-curves, of which are straight lines, parabolas and one branch right-angle hyperbolas. He also showed that geometrically inverted $\alpha$-curves, which are called by him $\beta$-curves support also invertible Radon transforms. The $\beta$-curves contains as special cases circles passing through a fixed point, cardioids and one-branch Bernoulli lemniscates. Palamodov has given the reconstruction of functions from the data of its integrals over half-circles with centers at the diameter of a half-disc $H$ [18]. But, as far as we are aware of, our circular arc Radon transform seems to be a new member of invertible Radon transforms in the plane.

Relations between these algebraic curve Radon transforms with the classical Radon have been routinely studied. In particular, the connection between parabolic and straightline Radon transforms has been found by Denecker et. al. [19], whereas the relation between standard Radon transforms and SAR/SONAR transforms is discussed in [6]. 
In this paper, we shall be concerned essentially with the new Radon transform on arcs of circles and the derivation of its inverse. In section 2, we recall the principle of Compton scattering tomography as suggested more than thirty years back. We then review the modality proposed by S J Norton, who has shown that a possible implementation of Compton scattering tomography can be made with a circular Radon transform based on circles which pass through the radiation point source. We then introduce the new circular-arc Radon transform after showing how it occurs in a new modality of Compton scattering tomography. The merits of this circular-arc Radon transform cannot be claimed until the existence of its inverse transform is established. Section 3 is precisely devoted to the derivation of the inverse transform. This implies that image reconstruction is feasible with Compton scattered radiation in this modality of Compton scattering tomography. Finally section 4 discusses some possible extensions of this Radon transform on families of algebraic curves globally invariant under geometric inversion. Conclusion and perspectives are contained in the last section.

\section{Compton scattering tomography (CST) and circular Radon Transforms}

\subsection{Compton scattering tomography (CST)}

For more than forty years, transmitted penetrating radiation such as X- or gamma-rays have been routinely used to probe the hidden parts of matter and/or tissues [20,21, 22]. The measurement of their attenuation along all possible linear paths in the plane form a set of Radon data, which, once fed into a chosen inversion formula provides the reconstruction of the probed medium. In this imaging modality radiation scatter acts as a nuisance by blurring images and it should be removed or at least be compensated.

However it was realized, in the earlier seventies, that the Compton effect which is the scattering of $\mathrm{X}$ - or gamma-photons with electric charges in matter may give rise to new challenging imaging modalities [23]. The idea is to register the outgoing scattered photons according to their energies in order to image the hidden part of objects of interest. Compton scattering tomography (CST) was then born. Several modalities have been proposed and tested [24]. But so far none of them has emerged as sufficiently efficient to be of widespread use.

Extension of this concept to three-dimensional imaging exists in two modalities: Compton camera imaging [25] and Compton scattered radiation imaging [26, 27, 28, 29]. The first one is based on Radon transform on conical surfaces with swinging axis whereas the second one is modeled by Radon transform on cone surfaces with fixed axis direction. Both of them are illustrations of an astute use of Compton scattering for imaging hidden parts of objects.

\subsection{Norton's $C T$}

In $1984 \mathrm{~S} \mathrm{~J} \mathrm{Norton} \mathrm{[10,30]} \mathrm{worked} \mathrm{out} \mathrm{a} \mathrm{CST} \mathrm{modality} \mathrm{which} \mathrm{is} \mathrm{based} \mathrm{on} \mathrm{a} \mathrm{Radon} \mathrm{transform}$ on circles having a fixed common point. The functioning principle is given by figure 1. A point source $\mathbf{S}$ emits primary radiation towards an object, of which $\mathbf{M}$ is a running point. A point detector $\mathbf{D}$ moves along an $O x$-axis and collects, at given energy $E$, scattered radiation from the object. The physics of Compton scattering demand that the registered radiation flux energy $\widehat{f}$ at site $\mathbf{D}$ is due to the contribution of all scattering sites $\mathbf{M}$ lying on an arc of circle from $\mathbf{S}$ to $\mathbf{D}$ subtending an angle $(\pi-\omega)$, where $\omega$ is the scattering angle corresponding to the outgoing energy $E$, as given by the Compton formula, see for 


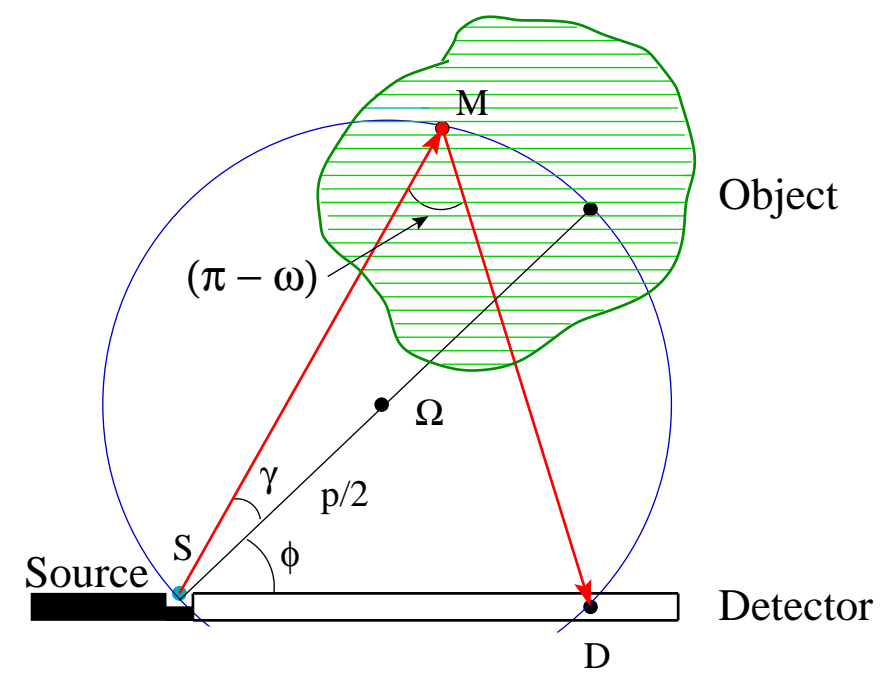

Figure 1. Norton's Compton scatter tomography

example [31]. Mathematically, $\widehat{f}$ is essentially the Radon transform of the object electron density $f(\mathbf{M})$ on such arc of circles, when radiation attenuation and photometric effects on radiation propagation are neglected. Image reconstruction is then feasible if an exact inversion formula for this type of circular Radon transform is available. This was done first by A M Cormack with a new technique of inversion, which turned out to work also for $\alpha$ - and $\beta$-curves, see [12]. Much later on, Norton came up with an alternative inversion formula [10].

\subsection{Review of the Cormack circular Radon transform inverse}

To facilitate the reading of the next sections, we briefly recall the inversion approach to the Cormack circular Radon transform, introduced above.

Consider a circle of diameter $p$, which goes through $S$ the origin of coordinates, see figure 1. Let its center $\Omega$ be defined by $\overrightarrow{S \Omega}=(p / 2) \mathbf{n}$, such that $\mathbf{n}$ makes an angle $\phi$ with respect to a reference direction $S x$. A running point $M$ on the circle is defined by $\overrightarrow{S M}=r(\gamma) \mathbf{k}$, where $\cos \gamma=(\mathbf{k} \cdot \mathbf{n})$. The polar equation of the circle is clearly $\cos \gamma=r / p$, with $-\pi / 2<\gamma<\pi / 2$. The circle arc element can be easily evaluated as

$$
d s=\sqrt{(d r)^{2}+(r d \gamma)^{2}}=r d \gamma \frac{1}{\cos \gamma}=p d \gamma
$$

An integrable function $f(\mathbf{M})$ is represented, in this polar coordinate system by $f(r, \theta)$, where $\theta=\gamma+\phi$. Its circular Radon transform is given by the integral

$$
\widehat{f}(p, \phi)=\left.\int_{\text {Arc }} d s f(r, \gamma+\phi)\right|_{r=p \cos \gamma}=\left.p \int_{-\pi / 2}^{\pi / 2} d \gamma f(r, \gamma+\phi)\right|_{r=p \cos \gamma},
$$

This defining equation can be reformulated in terms of Fourier angular components of $f$ and $\widehat{f}$

$$
f(r, \theta)=\sum_{l} f_{l}(r) e^{i l \theta}, \text { with } f_{l}(r)=\frac{1}{2 \pi} \int_{0}^{2 \pi} d \theta e^{-i l \theta} f(r, \theta)
$$




$$
\widehat{f}(p, \phi)=\sum_{l} \widehat{f}_{l}(p) e^{i l \phi} \text {, with } \widehat{f}_{l}(p)=\frac{1}{2 \pi} \int_{0}^{2 \pi} d \phi e^{-i l \phi} \widehat{f}(p, \phi)
$$

as

$$
\widehat{f}_{l}(p)=\left.p \int_{-\pi / 2}^{\pi / 2} d \gamma f_{l}(r)\right|_{r=p \cos \gamma} e^{i l \gamma} .
$$

From the polar equation of the circle we have

$$
r d \gamma \frac{1}{\cos \gamma}=p d \gamma=-\frac{d r}{\sin \gamma}
$$

and taking into account the symmetry in $\gamma$, a final form, after going back to the variable $r$, change of integration bounds, and expressing all quantities in terms of $r$, arises as

$$
\widehat{f}_{l}(p)=2 \int_{0}^{p} d r \frac{\cos l\left(\cos ^{-1}(r / p)\right)}{\sqrt{1-(r / p)^{2}}} f_{l}(r) .
$$

This is in fact one of the forms of the Tchebycheff transform [32], called Cormack transform, by H H Barrett in [33]. The recovery of the circular component $f_{l}(r)$ is done by integrating both sides of this equation on an appropriately chosen function of $p$. Thanks to a formula discovered by A M Cormack [12] (which is in fact a special case of a general property of Gauss hypergeometric functions [34]), the right-hand-side of the equation turns out to be the primitive of the product of $f_{l}(r)$ with a known function of $r$. Thus $f_{l}(r)$ can be extracted by a simple derivation. We shall meet the same inversion procedure for the circular arc Radon transform.

\subsection{A new modality in CST}

Recently we have suggested a new modality for Compton scattering tomography and presented some preliminary results on its performance [11]. Figure 2 shows how this novel modality of Compton scattering tomography works. An emitting radiation point source $\mathbf{S}$ is placed at a distance $2 p$ from a point detector $\mathbf{D}$. The segment $S D$ joining them rotates around its middle point $\mathbf{O}$. At site $\mathbf{D}$ is collected the single-scattered radiation flux density from the scanned object for a given angular position of the line $S D$ and at a given scattering energy $E$, (or equivalently at scattering angle $\omega$ ). Thus, thanks to the physics of the Compton effect, the detected radiation flux density $\widehat{f}(\tau, \varphi)$ is the integral of the electron density $f(\mathbf{M})$ on this class of circular arc, where $\tau=\cot \omega$ and $\varphi$ is the rotation angle made by the mediator line of the segment $S D$ with a fixed reference axis, see figure 2. Consequently this image formation leads to a novel class of Radon transform on a particular class of circular arcs, provided that radiation attenuation and photometric effects on radiation propagation are not taken into account.

\subsection{Circular-Arc Radon (CAR) transform}

The family of circular arcs, on which this new Radon transform is defined, subtend an angle $(\pi-\omega)$. Their radius is $p / \sin \omega$. A running point $M$ on the circular arc, is localized by its polar angle $\theta$ and its distance to the origin $O M=r$. The direction of $O M$ makes an angle $\gamma$ with the mediator line of $S D$, which itself makes an angle $\varphi$ with a fixed polar direction, as shown in figure 2.

From the cosine identity for the triangle $\Omega O M$

$$
\Omega M^{2}=O M^{2}+O \Omega^{2}-2 O M O \Omega \cos \widehat{\Omega O M},
$$




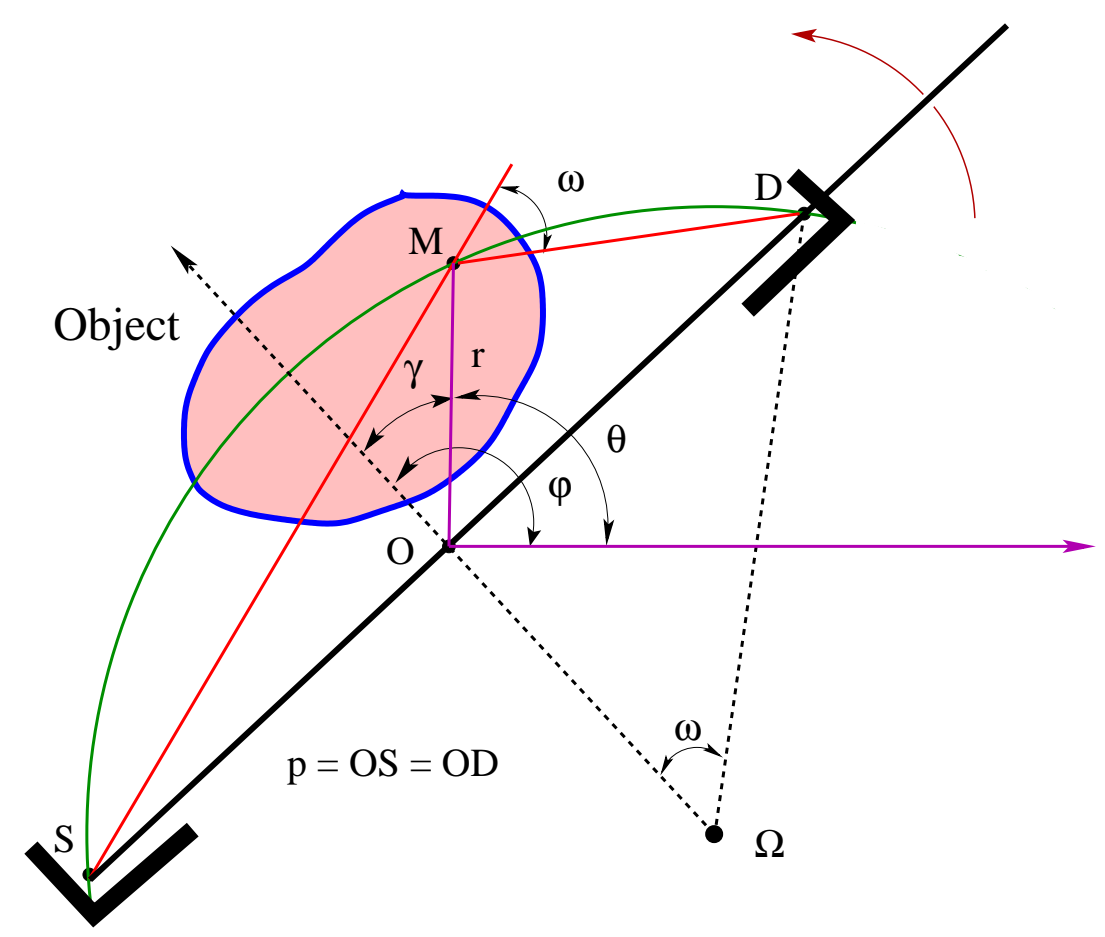

Figure 2. Principle of the Circular-Arc-Radon transform

and one can obtain the circle equation after making the substitutions $\Omega M=p / \sin \omega$, 
$O M=r$ and $\widehat{\Omega O M}=(\pi-\gamma)$ in the previous equation,

$$
p^{2}=r^{2}+2 p r \tau \cos \gamma
$$

The $r$-positive root of eq. (8) describes the physically relevant circular arc

$$
r=r(\cos \gamma)=p\left(\sqrt{1+\tau^{2} \cos ^{2} \gamma}-\tau \cos \gamma\right) .
$$

The second $r$-root describes the complementary arc below the $S D$ line, which corresponds to a subtended angle $\omega$, hence physically not relevant. As the product of the $r$-roots of eq. (8) is $p^{2}$, these two arcs are inverse from each other in a geometric inversion transform of center $O$ and module $p$. Consequently the whole circle is globally invariant with respect to this inversion and the locus of invariant points in this inversion is the circle $(\Gamma)$ of radius $p$ and center $O$. Thus the physical range of $\gamma$ is $-\pi / 2<\gamma<\pi / 2$, and $p\left(\sqrt{1+\tau^{2}}-\tau\right)<r<p$. Outside this interval $r=0$.

Alternatively, the circular-arc equation may also be written as

$$
\cos \gamma=\frac{1}{2 \tau}\left(\frac{p}{r}-\frac{r}{p}\right)
$$

displaying a reflection symmetry with respect to $\gamma$. In fact the symmetry is even greater, as the equation remains invariant under the simultaneous transformations

$$
\omega \longrightarrow(\pi-\omega) \text { and }(r, p) \longrightarrow(1 / r, 1 / p) .
$$

Actually we do not need back scattering because by rotating the scanning equipment beyond $\varphi>\pi$, we can find ourselves in the same situation as exploring with an angle larger than $\pi / 2$. This fact can be also seen in the inversion equation. Replacing $\omega$ by $(\pi-\omega)$ amounts to flip the sign of $\tau$. This is also equivalent to take the equation

$$
\cos \gamma=\frac{1}{2 \tau}\left(\frac{p}{r}+\frac{r}{p}\right)
$$

instead of equation (10). The circular-arc line element $d s$ can be readily given as

$$
d s=r d \gamma \sqrt{\frac{1+\tau^{2}}{1+\tau^{2} \cos ^{2} \gamma}}=d r \frac{\sqrt{1+\tau^{2}}}{\tau \sin \gamma} .
$$

With this line element, the Circular-Arc Radon (CAR)transform of a function $f(r, \theta)$, is

$$
\widehat{f}(\tau, \varphi)=\int_{\widehat{S D}} d s f(r, \theta),
$$

in which $f$ is an integrable function having a compact support, which is assumed to be strictly inside the inversion circle $(\Gamma)$.

We now derive a new integral equation linking $\widehat{f}_{l}(\tau)$ to $f_{l}(r)$, the circular components of $\widehat{f}(\tau, \varphi)$ and $f(r, \theta)$. Since $\theta=(\varphi-\gamma)$ from figure 2 and accounting for the invariance of the integrand under $\gamma \longleftrightarrow-\gamma$, eq. (13) takes the form

$$
\widehat{f}_{l}(\tau)=2 \int_{0}^{\pi / 2} d \gamma r(\cos \gamma) \sqrt{\frac{1+\tau^{2}}{1+\tau^{2} \cos ^{2} \gamma}} f_{l}(r(\cos \gamma)) \cos l \gamma .
$$

Now using

$$
d \gamma \frac{r(\cos \gamma)}{\sqrt{1+\tau^{2} \cos ^{2} \gamma}}=\frac{d r}{\tau \sin \gamma}=\frac{d r}{\sqrt{\tau^{2}-\frac{1}{4}\left(\frac{p}{r}-\frac{r}{p}\right)^{2}}}
$$


we change back to the $r$-variable in eq. (14) and compute the new $r$-integration bounds

$$
\begin{array}{ll}
\text { for } \gamma=0, & r=p\left(\sqrt{1+\tau^{2}}-\tau\right), \\
\text { for } \gamma=\pi / 2, & r=p .
\end{array}
$$

Eq. (14) becomes finally

$$
\frac{\tau \widehat{f}_{l}(\tau)}{\sqrt{1+\tau^{2}}}=2 \int_{p\left(\sqrt{1+\tau^{2}}-\tau\right)}^{p} \frac{d r}{\sqrt{1-\frac{1}{4 \tau^{2}}\left(\frac{p}{r}-\frac{r}{p}\right)^{2}}} f_{l}(r) \cos \left[l \cos ^{-1}\left(\frac{1}{2 \tau}\left(\frac{p}{r}-\frac{r}{p}\right)\right)\right] .
$$

Compare this equation (18) to equation (6).

\section{Inversion of the Circular-Arc-Radon Transform}

\subsection{Derivation}

We are now in a position to put equation (18) in the form of a Cormack's integral [12], which lends itself to inversion using an integral identity [34]. Let

$$
q=\frac{1}{\tau}=\tan \omega, \text { and } s^{-1}=\frac{1}{2}\left(\frac{p}{r}-\frac{r}{p}\right) .
$$

Since $r>0$, we have $r=p\left(\sqrt{1+s^{-2}}-s^{-1}\right)$, and

$$
\frac{d r}{r}=\frac{d s}{s \sqrt{1+s^{2}}} \text {. }
$$

For simplicity, let $h_{l}(r)=r f_{l}(r)$. The new integration boundary values on $s$ are

$$
\begin{array}{ll}
\text { for } r=p\left(\sqrt{1+\tau^{2}}-\tau\right), & s=q \\
\text { for } r=p, & s=\infty .
\end{array}
$$

Moreover with

$$
\frac{\tau}{\sqrt{1+\tau^{2}}}=\frac{1}{\sqrt{1+q^{2}}}
$$

eq. (18) becomes

$$
\frac{\widehat{f}_{l}(1 / q)}{\sqrt{1+q^{2}}}=2 \int_{q}^{\infty} \frac{d s}{s \sqrt{1+s^{2}}} h_{l}\left(p\left(\sqrt{1+s^{-2}}-s^{-1}\right)\right) \frac{\cos l\left(\cos ^{-1}(q / s)\right)}{\sqrt{1-(q / s)^{2}}} .
$$

This equation (24) has now exactly the structure of equation (12a) of [12] and becomes appropriate for Cormack's inversion technique. We follow Cormack's procedure by multiplying both sides of equation (24) by

$$
\frac{\cosh \left(l \cosh ^{-1}(q / t)\right)}{q \sqrt{(q / t)^{2}-1}}
$$

and integrate over $q$ from $t$ to $\infty$. This yields

$$
\begin{gathered}
\int_{t}^{\infty} d q \frac{\cosh \left(l \cosh ^{-1}(q / t)\right)}{q \sqrt{(q / t)^{2}-1}} \frac{\widehat{f}_{l}(1 / q)}{\sqrt{1+q^{2}}}= \\
2 \int_{t}^{\infty} d q \frac{\cosh \left(l \cosh ^{-1}(q / t)\right)}{q \sqrt{(q / t)^{2}-1}} \int_{q}^{\infty} \frac{d s}{s \sqrt{1+s^{2}}} h_{l}\left(p\left(\sqrt{1+s^{-2}}-s^{-1}\right)\right) \frac{\cos l\left(\cos ^{-1}(q / s)\right)}{\sqrt{1-(q / s)^{2}}} .
\end{gathered}
$$


Since $f_{l}(r)$ is of compact support, we can rearrange the two-dimensional integration on the right-hand-side of eq. (26) into

$2 \int_{t}^{\infty} \frac{d s}{s \sqrt{1+s^{2}}} h_{l}\left(p\left(\sqrt{1+s^{-2}}-s^{-1}\right)\right) \int_{t}^{s} d q \frac{\cosh \left(l \cosh ^{-1}(q / t)\right)}{q \sqrt{(q / t)^{2}-1}} \frac{\cos l\left(\cos ^{-1}(q / s)\right)}{\sqrt{1-(q / s)^{2}}}$.

But in $[12,34]$, it is shown that

$$
\int_{t}^{s} d q \frac{\cosh \left(l \cosh ^{-1}\left(\frac{q}{t}\right)\right)}{q \sqrt{\left(\frac{q}{t}\right)^{2}-1}} \frac{\cos l\left(\cos ^{-1}(q / s)\right)}{\sqrt{1-(q / s)^{2}}}=\frac{\pi}{2} .
$$

This result leads to the following form of the integral relation between circular components

$$
\int_{t}^{\infty} \frac{d s}{s \sqrt{1+s^{2}}} h_{l}\left(p\left(\sqrt{1+s^{-2}}-s^{-1}\right)\right)=\frac{1}{\pi} \int_{t}^{\infty} d q \frac{\cosh \left(l \cosh ^{-1}(q / t)\right)}{q \sqrt{(q / t)^{2}-1}} \frac{\widehat{f}_{l}(1 / q)}{\sqrt{1+q^{2}}}
$$

Extraction of $f_{l}$ is achieved by differentiation with respect to $t$ on both sides of eq. (29)

$$
\begin{gathered}
\frac{-\pi}{t \sqrt{1+t^{2}}} p\left(\sqrt{1+t^{-2}}-t^{-1}\right) f_{l}\left(p\left(\sqrt{1+s^{-2}}-s^{-1}\right)\right)= \\
{\left[\frac{d}{d t} \int_{t}^{\infty} d q \frac{\cosh \left(l \cosh ^{-1}(q / t)\right)}{q \sqrt{(q / t)^{2}-1}} \frac{\widehat{f}_{l}(1 / q)}{\sqrt{1+q^{2}}}\right]_{\left.t=\frac{2 p r}{\left(p^{2}-r^{2}\right.}\right)} .}
\end{gathered}
$$

It remains now to re-insert the original variable $r$ in eq. (30). Recalling that, by definition, $r=p\left(\sqrt{1+t^{-2}}-t^{-1}\right)$, we can work out

$$
t=\frac{2 p r}{\left(p^{2}-r^{2}\right)}, \text { and } \frac{1}{t \sqrt{1+t^{2}}}=\frac{2 p r}{\left(p^{2}+r^{2}\right)} .
$$

Eq. (30) yields the circular component $f_{l}(r)$ of $f(r, \theta)$

$$
f_{l}(r)=(-) \frac{\left(p^{2}+r^{2}\right)}{2 \pi p r^{2}}\left[\frac{d}{d t} \int_{t}^{\infty} d q \frac{\cosh \left(l \cosh ^{-1}(q / t)\right)}{q \sqrt{(q / t)^{2}-1}} \frac{\widehat{f}_{l}(1 / q)}{\sqrt{1+q^{2}}}\right]_{\left.t=\frac{2 p r}{\left(p^{2}-r^{2}\right.}\right)} .
$$

A simple change of variables in the integration shows that equation (32) may be recast as

$$
f_{l}(r)=(-) \frac{\left(p^{2}+r^{2}\right)}{2 \pi p r^{2}}\left[\int_{t}^{\infty} d q \frac{\cosh \left(l \cosh ^{-1}(q / t)\right)}{\sqrt{q^{2}-t^{2}}} \frac{d}{d q}\left(\frac{\widehat{f}_{l}(1 / q)}{\sqrt{1+q^{2}}}\right)\right]_{\left.t=\frac{2 p r}{\left(p^{2}-r^{2}\right.}\right)} .
$$

Finally $f(r, \theta)$ is reconstructed through its Fourier expansion with the circular components $f_{l}(r)$, as given by eq. (32). Note that the integration on $q$ means that one has to collect data with scattering angle from $\pi / 2$ to a certain value corresponding to $t$. This is the hole theorem as quoted in [12]. Finally this inversion structure suffers from data noise propagation for which a cure is proposed in [35]. 


\subsection{Advantages of the new CST modality}

The analytical inverse formula (32) (or (33)) is the mathematical basis for a new image reconstruction method via the object electron density in this CST modality. This may be viewed as an alternative to image reconstruction via the object attenuation map by standard computed tomography (CT), because the attenuation property of matter may change in time as opposed to its electron density. It opens a new way for probing the inside of matter under working conditions which complement those of the existing CST modality. In fact in Norton's 1995 CST modality, the nature of the circular Radon transform dictates a scanning process on one side of the line source - detector. This is appropriate for large objects such as concrete walls, metal structures in ship building and the like. However there exists also a need for imaging smaller objects in non-destructive industrial testing as well as in medical imaging. For these objects, scanning should be rapid and restricted to an adjustable reduced volume in which residual stray radiation can be easily shielded. This is why the proposed CST modality, based on the circular-arc Radon transform, is more appropriate, in particular when a large number of objects need to be imaged in a row. Moreover, such a CST scanner can be built with the existing detector/detection technology, which has been shown to work with primary (or non-scattered radiation) as well as with scattered radiation. This would make it suitable for widespread use.

\section{A generalization of the circular-arc Radon transform}

\subsection{Definition}

In this section we examine the question of whether or not one can extend the idea of Cormack for finding more general curves on which a Radon transform can be defined so that it can be inverted by the same procedure. The essence of the idea is to come up with a similar functional form by assuming at first that relevant curves should be of the general type

$$
\tau \cos (a \gamma)=g\left(x^{a}\right), \text { where } x=\frac{p}{r},-\frac{\pi}{2 a}<\gamma<\frac{\pi}{2 a} \text { and } a \in \mathbb{R}^{+} .
$$

Here $g$ is an arbitrary function, $p$ a characteristic length of the system and $\tau \in \mathbb{R}^{+}$a parameter. By differentiation one can show that

$$
\frac{r d \gamma}{x^{a} g^{\prime}\left(x^{a}\right)}=\frac{d r}{\tau \sin (a \gamma)}=\frac{d r}{\sqrt{\tau^{2}-g^{2}\left(x^{a}\right)}} .
$$

Hence the arc element is

$$
d s=\sqrt{d r^{2}+(r d \gamma)^{2}}=d r \sqrt{\frac{\tau^{2}-g^{2}\left(x^{a}\right)+\left(x^{a} g^{\prime}\left(x^{a}\right)\right)^{2}}{\tau^{2}-g^{2}\left(x^{a}\right)}} .
$$

This allows to define the Radon transform of a function $f(r, \theta)$ as

$$
\widehat{f}(\tau, \varphi)=\int_{\widehat{S D}} d s f(r(\gamma), \gamma+\varphi)=\int_{r_{1}}^{r_{2}} d r \sqrt{\frac{\tau^{2}-g^{2}\left(x^{a}\right)+\left(x^{a} g^{\prime}\left(x^{a}\right)\right)^{2}}{\tau^{2}-g^{2}\left(x^{a}\right)}} f(r, \gamma+\varphi),
$$

where $\gamma=a^{-1} \cos ^{-1}(g / \tau)$. The integration limits are determined by $g\left(\left(p / r_{1}\right)^{a}\right)=\tau$ and by $g\left(\left(p / r_{2}\right)^{a}\right)=0$. Now going to the circular components as before, the integral equation linking them is

$$
\widehat{f}_{l}(\tau)=\left.2 \int_{r_{1}}^{r_{2}} d r \sqrt{\frac{\tau^{2}-g^{2}\left(x^{a}\right)+\left(x^{a} g^{\prime}\left(x^{a}\right)\right)^{2}}{\tau^{2}-g^{2}\left(x^{a}\right)}} f_{l}(r) \cos (l \gamma)\right|_{\gamma=a^{-1} \cos ^{-1}(g / \tau)} .
$$


Since $g$ does not depend on $\tau$, if we demand that

$$
-g^{2}\left(x^{a}\right)+\left(x^{a} g^{\prime}\left(x^{a}\right)\right)^{2}=C \text { constant, }
$$

then eq. (38) becomes

$$
\frac{\tau}{\sqrt{\tau^{2}+C}} \widehat{f}_{l}(\tau)=2 \int_{r_{1}}^{r_{2}} d r \frac{\cos \left(l / a \cos ^{-1}(g / \tau)\right)}{\sqrt{1-\left(g\left(x^{a}\right) / \tau\right)^{2}}} f_{l}(r) .
$$

This equation has the required form for inversion with the Cormack's method.

So we must solve the differential equation (39). Putting $z=\left(x^{a}\right)$ with $x=p / r$, it is easy to verify that the general solution takes the form of a linear fractional relation

$$
g(z)=\left(p z+\frac{q}{z}\right) \text { with } C=-4 p q .
$$

Conversely we can work out $z$ (resp. $r$ ) as a function of $g$

$$
z=\frac{g}{2 p} \pm \sqrt{\left(\frac{g}{2 p}\right)^{2}-\frac{q}{p}} \text { and } r=p\left(\frac{g}{2 p} \pm \sqrt{\left(\frac{g}{2 p}\right)^{2}-\frac{q}{p}}\right)^{1 / a},
$$

and compute $d r$ in terms of $g$, and replace $r$ by its expression in $g$.

\section{Remark}

A different type of generalization has been studied by Kurusa [36]. It is defined on closed curves of $\mathbb{R}^{2}$ having strictly convex distance functions.

\subsection{Special cases}

We observe that eq. (41) contains the two cases solved by A M Cormack when either $p$ or $q$ is zero, i.e.

$$
\cos a \gamma=\left(\frac{p}{r}\right)^{a} \text { for } q=0, \quad \text { and } \cos a \gamma=\left(\frac{r}{q}\right)^{a} \text { for } p=0
$$

We now examine a non-trivial example of the previous generalization, for which the curve equation is simply

$$
\cos a \gamma=\frac{1}{2 \tau}\left[\left(\frac{p}{r}\right)^{a}-\left(\frac{r}{p}\right)^{a}\right],
$$

where $a \in \mathbb{R}^{+}$and $-\pi / 2 a<\gamma<\pi / 2 a$. Alternatively, we can solve for $r$

$$
\left(\frac{r}{p}\right)^{a}=\left(\sqrt{1+\tau^{2} \cos ^{2} a \gamma}-\tau \cos a \gamma\right)
$$

For $a=1$, the curve is a circular arc $\overparen{S D}$ rotating around a point $O$, which is no longer the middle point of the chord $S D$. The corresponding differential $d r$ is

$$
d r=\frac{\tau \sin a \gamma}{\sqrt{1+\tau^{2} \cos ^{2} a \gamma}} r(\cos \gamma) d \gamma
$$

hence the curve element $d s$ is

$$
d s=r d \gamma \sqrt{\frac{1+\tau^{2}}{1+\tau^{2} \cos ^{2} a \gamma}}=d r \frac{1}{\tau \sin a \gamma} .
$$

The curvilinear-arc Radon transform is now given by the integral

$$
\widehat{f}(\tau, \varphi)=\int_{\text {Arc }} d s f(r(\cos \gamma), \theta)=\int_{-\pi / 2 a}^{\pi / 2 a} r d \gamma \sqrt{\frac{1+\tau^{2}}{1+\tau^{2} \cos ^{2} a \gamma}} f(r(\cos \gamma), \gamma+\varphi) .
$$


Going over circular components we find

$$
\widehat{f}_{l}(\tau)=2 \int_{-\pi / 2 a}^{\pi / 2 a} r(\cos \gamma) d \gamma \sqrt{\frac{1+\tau^{2}}{1+\tau^{2} \cos ^{2} a \gamma}} f_{l}(r(\cos \gamma)) \cos (l \gamma)
$$

Now after going back to the $r$-variable, this integral equation becomes

$$
\frac{\tau \widehat{f}_{l}(\tau)}{\sqrt{1+\tau^{2}}}=2 \int_{p\left(\sqrt{1+\tau^{2}}-\tau\right)^{1 / a}}^{p} \frac{d r}{\sqrt{1-\left(\frac{1}{2 \tau}\left[\left(\frac{p}{r}\right)^{a}-\left(\frac{r}{p}\right)^{a}\right]\right)^{2}}} f_{l}(r) \cos (l \gamma) .
$$

with

$$
\gamma=\frac{1}{a} \cos ^{-1} \frac{1}{2 \tau}\left[\left(\frac{p}{r}\right)^{a}-\left(\frac{r}{p}\right)^{a}\right]
$$

Setting as before

$$
q=\frac{1}{\tau} \text { and } \frac{1}{s}=\frac{1}{2}\left[\left(\frac{p}{r}\right)^{a}-\left(\frac{r}{p}\right)^{a}\right] \Rightarrow d r=\frac{r}{a} \frac{d s}{s \sqrt{1+s^{2}}},
$$

we end up with the final equation

$$
\frac{\tau \widehat{f}_{l}(\tau)}{\sqrt{1+\tau^{2}}}=2 \int_{q}^{\infty} \frac{1}{a} \frac{d s}{s \sqrt{1+s^{2}}} \frac{1}{\sqrt{1-(q / s)^{2}}} h_{l}\left(p\left(\sqrt{1+s^{-2}}-(1 / s)\right)^{1 / a}\right) \cos \left(\frac{l}{a} \cos ^{-1}(q / s)\right)
$$

where $h_{l}(r)=r f_{l}(r)$ as before. This last equation has again the precise structure of Cormack's equation [12], thus invertible with the same technique.

In particular, for $a=1 / 2,2$, we have the equivalent inversion invariant $\alpha$ - and $\beta$ curves of [12].

- for $a=1 / 2$

$$
\sqrt{\frac{r}{p}}=\left(\sqrt{1+\tau^{2} \cos ^{2}(\gamma / 2)}-\tau \cos (\gamma / 2)\right)
$$

This curve is closed since $-\pi<\gamma<\pi$. It has the form of a one branch lemniscate.

- for $a=2$,

$$
\left(\frac{r}{p}\right)^{2}=\left(\sqrt{1+\tau^{2} \cos ^{2} 2 \gamma}-\tau \cos 2 \gamma\right) .
$$

This is an arc located in a $\pi / 2$-quadrant of the plane since $-\pi / 4<\gamma<\pi / 4$.

\subsection{Inclusion of attenuation and photometric effects in realistic working conditions}

For $a=1$, under realistic working conditions, traveling radiation is affected by medium attenuation and by dispersion due to photometric propagation effects. A standard way to take into account for these effects is to put the following factor

$$
\frac{e^{-\mu M S}}{M S^{2}} \times \frac{e^{-\mu M D}}{M D^{2}}
$$

(where $\mu$ is the average linear attenuation coefficient, here assumed to be constant) in the integrand of the circular-arc Radon transform of eq. (13). The value of this additional factor can be evaluated by using triangular identities

$$
M S^{2}=p^{2}+r^{2}-2 p r \sin \gamma \text { and } M D^{2}=p^{2}+r^{2}+2 p r \sin \gamma .
$$


Hence

$$
\begin{aligned}
\frac{e^{-\mu M S}}{M S^{2}} \times \frac{e^{-\mu M D}}{M D^{2}} & =\frac{\exp -\mu\left(\sqrt{p^{2}+r^{2}-2 p r \sin \gamma}+\sqrt{p^{2}+r^{2}+2 p r \sin \gamma}\right)}{\left(p^{2}-r^{2}\right)^{2}+4 p^{2} r^{2} \cos ^{2} \gamma} \\
& =\frac{\exp -\mu\left(\sqrt{p^{2}+r^{2}-2 p r \sin \gamma}+\sqrt{p^{2}+r^{2}+2 p r \sin \gamma}\right)}{\left(p^{2}-r^{2}\right)^{2}\left(1+\tau^{-2}\right)}
\end{aligned}
$$

where

$$
\sin \gamma=\sqrt{1-\left(\frac{1}{2 \tau}\left(\frac{p}{r}-\frac{r}{p}\right)\right)^{2}}>0,
$$

We observe that attenuation brings up a term in $\sin \gamma$, which has a non-separable dependence on $\tau$. Thus inversion cannot be achieved in this case with the present method. However the inclusion of photometric effects, which yields terms of the form of a product of a function of $r$ and a function of $\tau$, will not spoiled the present mechanism of inversion. These terms have apparent divergences at $r=p$, which are in fact avoided since the class of functions of interest have their support strictly inside the inversion circle $(\Gamma)$.

\section{Conclusion and perspectives}

A new circular arc Radon transform arising in the mathematical modeling of image formation of a new modality of Compton scatter tomography (CST) is introduced and shown to be invertible via the technique of A M Cormack. It is also proved that it is a member of a larger class of inversion invariant algebraic curve Radon transforms. Interesting mathematical problems can be raised in this context, such as questions on range and injectivity in general, which has been studied in depth for previous classes of circular Radon transforms [9, 37, 38]. Another aspect is the development of numerical reconstruction algorithms based on an adapted filtered back-projection process, which remains to be constructed and exploited. These topics could be the subjects for future work which can include the study of an extension to higher dimensional spaces as fascinating new invertible spherical Radon transforms of potential use for imaging science.

\section{References}

[1] Radon J 1917 Über die Bestimmung von Funktionnen durch ihre Integralwerte längs gewisser Mannigfaltikeiten Ber.Verh.Sachs.Akad.Wiss. Leipzig-Math.-Natur.Kl. 69 262-277.

[2] Gelfand I M 1960 Integral geometry and its relations to the theory of group representation Russian Math. Surveys 15 143-151.

[3] Quinto E T 1994 Radon transforms on curves in the plane Lectures in Applied Mathematics: Tomography, Impedance Imaging and Integral Geometry 30 231-244.

[4] Norton S J 1980 Reconstruction of a two-dimensional reflecting medium over a circular domain: exact solution, J. Acoust. Soc. Am. 64, 1266-1273.

[5] Finch D, Patch S K and Rakesh 2004 Determining a function from its mean values over a family of spheres, SIAM J. Math. Anal 35(5), 1213-1240.

[6] Redding N J and Newsam G N 2001 Inverting the circular Radon Transform, DSTO Electronics and Surveillance Research Laboratory Report DSTO-RR-0211, Edinburgh, Australia. http://www.dsto.defence.gov.au/corporate/reports/DSTO-RR-0211.pdf

[7] Sysoev S E 1997 Unique recovery of a function integrable in a strip from its integral over circles centered on a fixed line Communications of the Moscow Mathematical Society, 846-847.

[8] Louis A K and Quinto E T 2000 Local Tomography Mathods in SONAR, in Surveys on solution methods for inverse problems Eds: D. Colton, H. W. Engl, A. K. Louis, J. McLaughlin and W. Rundell, Springer New-York, 147-154. 
[9] Agranovsky M and Quinto E T 1996 Injectivity sets for the Radon transform over circles and complete systems of radial functions J. Functional Analysis 139, 383-414.

[10] Norton S J 1994, Compton scattering tomography, J. Appl. Phys. 76,2007-2015.

[11] Nguyen M K, Driol C, Truong T T, Paindavoine M, Ginhac D, 2009 Nouvelle Tomographie Compton,Proceedings GRETSI September 2009, Dijon France.

[12] Cormack A M 1983 The Radon transform on a family of curves in the plane Proceedings of the American Mathematical Society 83(2), 325-330.

[13] Mensah S, Franceschini E and Lefebvre J P 2006 Near-field ultrasound mammography, La Revue du Traitement du Signal, 3 (3).

[14] Maeland E 1998 Focusing aspects of the parabolic Radon transform Geophysics 63, 1708-1715.

[15] Lissianoi S and Ponomarev I 1997, On the inversion of the geodesic Radon transform on the hyperbolic plane, Inverse problems, 13, 1053-1062.

[16] Maass P 1989 Wideband radar: the hyp-transform Inverse Problems 5, 849-857.

[17] Morvidone M, Nguyen M K, Truong T T and Zaidi H 2009 On the V-line Radon transform and its imaging applications Preprint UCP, October 2009.

[18] Paladomov V P 2000 Reconstruction from limited data of arc means The journal of Fourier Analysis and Applications 6(1) 25-42.

[19] Denecker K, Van Overloop J and Sommen F 1998 The general quadratic Radon transform Inverse Problems 14 615-633.

[20] Hussein E M A, Meneley D A and Banerjee S 1986 On the solution of the inverse problem of radiation scattering imaging, Nuclear Science and Engineering, 92, 341-349.

[21] Arendtsz N V and Husein E M A 1995 Energy-spectral Compton scatter Imaging - Part 1: theory and mathematics, IEEE Transactions on Nuclear Sciences 42(6), 2155-2165.

[22] Guzzardi R and Licitra G 1988 A critical review of Compton imaging, CRC Critical Reviews in Biomedical Imaging, 15(3), 237-268.

[23] Gerl J 2005 Gamma-ray imaging exploiting the Compton effect, Nuclear Physics, A 752, 688c-695c.

[24] Grangeat P 2002 La Tomographie (Hermès Science Paris).

[25] Singh M. 1983 An electronically collimated gamma camera for single photon emission computed tomography. Part 1: Theoretical considerations and design criteria Med. Phys., 10, 421-427.

[26] Nguyen M K and Truong T T 2002 On an integral transform and its inverse in nuclear imaging Inverse Problems 18 265-277.

[27] Nguyen M K, Truong T T, Bui H D and Delarbre J L 2004 A novel inverse problem in $\gamma$-rays emission imaging Inverse Problems in Science and in Engineering 12 (2) 225-246.

[28] Nguyen M K, Truong T T and Greangeat P 2005 Radon transforms on a class of cones with fixed axis direction Jour. Phys.A: Math. Gen.38 8003-8015.

[29] Nguyen M K, Truong T T, Driol C and Zaidi H, 2009 On a novel approach to Compton scattered emission imaging, IEEE Transactions in Nuclear Sciences, 56(3), 1430-1437.

[30] Wang J, Chi Z and Wang Y 1999 Analytic reconstruction of Compton scattering tomography, J. Appl. Phys. 86(3), 1693-1698.

[31] Barrett H H and Swindell W 1981 Radiological Imaging (Academic Press New York).

[32] Li T 1960 A new class of integral transforms, Proc. Amer. Math. Soc., 11, 290-298.

[33] Barrett H H 1984 The Radon Transform and its Applications in Progress in Optics 21 , pp. 219-286, Ed. E. Wolf, North Holland.

[34] Bushman R G Integrals of hypergeometric functions, Math. Zeitschr., 89, 74-76.

[35] Cormack A M 1984 Radon's problem - Old and new, SIAM - AMS Proceedings 14, 33-39.

[36] Kurusa A 1993 Support curves of invertible Radon transforms, Arch. Math., 61, 448-458.

[37] Ambartsoumian G and Kuchment P 2005 On the injectivity of the circular Radon transform Inverse Problems 21, 473-485.

[38] Ambartsoumian G and Kuchment P 2006 A range description for the planar circular Radon transform SIAM Journal on Mathematical Analysis 38(2), 681-692. 